\title{
Caspase-6 Induces 7A6 Antigen Localization to Mitochondria During FAS-induced Apoptosis of Jurkat Cells
}

\author{
HIROAKI SUITA, TAKAHISA SHINOMIYA and YUKITOSHI NAGAHARA \\ Division of Life Science and Engineering, School of Science and Engineering, \\ Tokyo Denki University, Hatoyama, Japan
}

\begin{abstract}
Background: Mitochondria are central to apoptosis. However, apoptosis progression involving mitochondria is not fully understood. A factor involved in mitochondria-mediated apoptosis is 7A6 antigen. 7A6 localizes to mitochondria from the cytosol during apoptosis, which seems to involve 'effector' caspases. In this study, we investigated the precise role of effector caspases in 7A6 localization to mitochondria during apoptosis. Materials and Methods: Human T-cell lymphoma Jurkat cells were treated with an antibody against FAS. 7A6 localization was analyzed by confocal laser scanning microscopy and flow cytometry. Caspases activation was determined by western blot analysis. Results: 7A6 localization to mitochondria during anti-FAS-induced apoptosis was significantly reduced by the caspase-6 inhibitor, $\mathrm{N}$-acetyl-Val-Glu-Ile-Asp-aldehyde, but not by the caspase-3 inhibitor, N-acetyl-Asp-Asn-Leu-Aspaldehyde, nor caspase-7/3 inhibitor, N-acetyl-Asp-Gln-ThrAsp-aldehyde. Moreover, caspase-6 down-regulation suppressed 7A6 localization to mitochondria. Conclusion: Caspase- 6 regulates $7 A 6$ localization to mitochondria during anti-FAS-induced apoptosis of Jurkat cells.
\end{abstract}

Caspases are important enzymes during apoptosis. They are cysteine proteases that contain cysteine as an active site. Apoptotic stimuli activate caspases, leading to substrate cleavage and resulting in apoptosis progression. To date, 14 caspases have been identified, and these have been divided into two major functional groups: one involves apoptosis progression (caspases 2, 3, 6, 7, 8, 9 and 10) and the other induces inflammation (caspases 1, 4, 5, 11 and 12) (1). Moreover, caspases involved in apoptosis are classified as either 'effector' caspases that execute apoptosis, or 'initiator'

Correspondence to: Yukitoshi Nagahara, Ph.D., Division of Life Science and Engineering, School of Science and Engineering, Tokyo Denki University, Hatoyama, Hiki-gun, Saitama, 350-0394 Japan. Tel: +81 492965949, Fax: +81 492965162, email: yuki@mail.dendai.ac.jp

Key Words: Apoptosis, Apo2.7, 7A6 antigen, caspase-6. caspases (caspase-8 and -9) that activate effector caspases (1). Caspases are constructed of a pro-domain, a large subunit, and a small subunit. Upon caspase activation, cleavage of the caspase occurs. Caspase-6 and -7, which are effector caspases, each have a large subunit that is $20 \mathrm{kDa}$ (p20) and a small subunit that is $10 \mathrm{kDa}$ (p10), but caspase-3, which is also an effector caspase, has a large subunit of $17 \mathrm{kDa}$ and a small subunit of $12 \mathrm{kDa}$ (p12) (2-4). Moreover, the large and small subunits of caspase- 6 and -7 are connected by a linker, but caspase-3 does not have a linker (Figure 1). The difference between effector and initiator caspases is that the pro-domain of an initiator caspase is long and that the pro-domain of an initiator caspase has a death effector domain or a caspase recruitment domain, but the effector caspase has neither (5).

Three effector caspases have short pro-domains, and are thought to induce apoptosis. Caspase- 3 and -7 are deeply involved in apoptosis, but the involvement of caspase- 6 is not known well (2).

Specific apoptotic stimuli activate different caspase activation pathways. One stimulus is a death receptor called FAS. Ligands that bind to FAS, FasL, and antibody to FAS induce the trimerization of FAS, leading to the activity of caspase-8, an initiator caspase, through FAS-associated protein with a death domain adapter protein (6). Then effector caspases, caspase-3 and -7, are activated, further cleaving many substrates, including poly (ADP-ribose) polymerase 1 (PARP1), fodrin gelsolin, vimentin and p21 (7). Caspase-6 is also activated by FAS and can cleave caspase-8. It was suggested that caspase- 6 plays a role in positive feedback of the apoptosis pathway (8).

Activated caspase- 8 also cleaves BH3 interacting domain death agonist (BID), a member of the B-cell lymphoma 2 (BCL2) family of proteins, into tBID, leading to mitochondrial damage and the release of cytochrome $c$ to the cytosol (9). The mitochondrion is a key organelle of the intrinsic apoptosis pathway. Mitochondria may be damaged directly by several chemicals, reactive oxygen species, and tBID $(9,10)$. Released cytochrome $c$ activates caspase-9 and further activates caspase-3, -7 , and $-6(9,11,12)$. Mitochondria can also release other apoptotic factors, 
including second mitochondria-derived activator of caspases (SMAC), high temperature requirement A2 (HTRA2), apoptosis-inducing factor (AIF), and endonuclease $G$ (EndoG) (13-15). Under the normal state, caspases are inhibited by inhibitors of apoptosis proteins (IAPs), thereby inhibiting apoptosis. When an apoptotic stimulus is applied to mitochondria, SMAC is released and degrades IAPs, leading to apoptosis. HTRA2 is similar to but more active than SMAC (13). AIF and EndoG directly damage DNA and induce apoptosis in a caspase-independent way $(14,15)$.

7A6 antigen is also a mitochondrial apoptosis-related protein. 7A6 was discovered by using Apo2.7, a monoclonal antibody, by immunizing mice with apoptotic human T-cell lymphoma Jurkat cells (16). 7A6 localizes to mitochondria at the early stages of apoptosis, as has been confirmed in many cell lines: T-cell lines Jurkat, Molt-4, CEM, and HUT-102; Bcell lines Daudi, Ramos, and Raji; histiocytic cell line U937; and promyelocytic cell line HL-60 (16). 7A6 can be induced by FAS, irradiation, DNA damage, drugs inducing mitochondrial injury, or viral infection (16). 7A6 has also been investigated in necrotic cells (17). However, it is possible to detect dead cells without their having undergone apoptosis. 7A6 localization is reported as being either caspase-dependent or -independent, depending on the study $(18,19)$.

In a previous study, anti-FAS as a death receptor stimulus, and FTY720, a mitochondria-damaging immunosuppressant, were each observed to induce caspase activation, DNA fragmentation, and 7A6 localization to mitochondria (18). However, preincubation with pan-caspase inhibitor benzyloxycarbonyl-ValAla-Asp (OMe) fluoromethylketone (z-VAD-fmk), or with caspase-3-specific inhibitor $N$-acetyl-Asp-Glu-Val-Asp-aldehyde (Ac-DEVD-CHO), inhibited all of the above, including 7A6 localization to mitochondria, suggesting that 7A6 localization to mitochondria occurs after caspase activation and is caspasedependent. However, DEVD, a caspase-3 inhibitor amino acid sequence, is not highly specific and also inhibits caspase- 6 and -7 (20). Thus, 7A6 localization might be the result of effector caspase activation. To identify the effector caspase involved in 7A6 localization to mitochondria, we used highly specific caspase inhibitors to seek the effect of each caspase on 7A6. Furthermore, we aimed to elucidate the role and function of 7A6 in caspase-dependent apoptosis.

\section{Materials and Methods}

Cell culture. Jurkat cells were provided by Dr. T. Miyashita of the National Research Institute for Child Health and Development (Tokyo, Japan) and cultured in RPMI-1640 medium supplemented with $10 \%$ fetal bovine serum (FBS) and $75 \mathrm{mg} / \mathrm{l}$ kanamycin sulfate, and maintained at $37^{\circ} \mathrm{C}$ in a humidified chamber under an atmosphere of $95 \%$ air and $5 \% \mathrm{CO}_{2}$.

Caspase-6 knockdown by siRNA electroporation. For electroporation with the Neon System, Jurkat cells were washed with PBS without $\mathrm{Ca}^{2+}$ and $\mathrm{Mg}^{2+}$ and centrifuged at $300 \times g$ for
3 min. Cells were pelleted and suspended in Resuspension Buffer $\mathrm{R}$ (Neon $10 \mu \mathrm{l}$ kit; ThermoFisher Scientific, Waltham, MA, USA) to a final density of $5 \times 10^{7}$ cells $/ \mathrm{ml}$ and then added to caspase- 6 specific siRNA or negative siRNA control, Control siRNA-A (Santa Cruz Biotechnology, Dallas, TX, USA). Both siRNA concentrations were $0.25 \mu \mathrm{M}$. Cells underwent siRNA transfection by electroporation (three $10 \mathrm{~ms}$ pulses of $1350 \mathrm{~V}$ ) and were then seeded in 24-well plates for $48 \mathrm{~h}$.

Determination of 7A6 antigen using confocal laser scanning microscopy. Cells $\left(2 \times 10^{6}\right)$ were treated with $100 \mathrm{nM}$ MitoTracker Green (ThermoFisher Scientific) at $37^{\circ} \mathrm{C}$ for $1 \mathrm{~h}$. Next, the cells were permeabilized with $20 \mu \mathrm{g} / \mathrm{ml}$ digitonin in $5 \%$ bovine serum albumin (BSA)-PBS for $20 \mathrm{~min}$ on ice. After incubation, cells were stained with R phycoerythrin-cyanin 5.1 (PC5)-labeled Apo2.7 (Beckman Coulter, Buckinghamshire, UK) for $60 \mathrm{~min}$. 7A6 antigen was observed by confocal laser scanning microscopy (FV10; Olympus, Tokyo, Japan).

Determination of 7A6 antigen using flow cytometry (FCM). After incubation in the presence or absence of $50 \mu \mathrm{M}$ caspase inhibitors [pan-caspase inhibitor benzyloxycarbonyl-Asp-2,6-dichlorobenzoyloxymethylketone (z-Asp- $\mathrm{CH}_{2}-\mathrm{DCB}$ ), caspase-3-specific inhibitor $\mathrm{N}$ acetyl-Asp-Asn-Leu-Asp-aldehyde (Ac-DNLD-CHO), caspase-7/3specific inhibitor $N$-acetyl-Asp-Gln-Thr-Asp-aldehyde (Ac-DQTD$\mathrm{CHO}$ ), and caspase-6-specific inhibitor $N$-acetyl-Val-Glu-Ile-Aspaldehyde (Ac-VEID-CHO)] for $1 \mathrm{~h}$ and further incubation with 0.5 $\mu \mathrm{g} / \mathrm{ml}$ anti-FAS for $6 \mathrm{~h}$, cells $\left(2 \times 10^{6}\right)$ were collected and permeabilized with $20 \mu \mathrm{g} / \mathrm{ml}$ digitonin in 5\% BSA-PBS for $20 \mathrm{~min}$ on ice. After incubation, cells were stained with PC5-labeled Apo2.7 for 60 min. 7A6 antigen was quantified by FCM (FACSCalibur; Becton Dickinson, Franklin Lakes, NJ, USA). Small fragmented particles and aggregates were excluded by gating, and the data were analyzed by CellQuest software (Becton Dickinson).

Agarose gel electrophoresis. Apoptosis was determined by DNA fragmentation, as assessed by agarose gel electrophoresis. After incubation in the presence or absence of $50 \mu \mathrm{M}$ caspase inhibitor (zAsp- $\mathrm{CH}_{2}$-DCB) for $1 \mathrm{~h}$ and further incubation with $0.5 \mu \mathrm{g} / \mathrm{ml}$ antiFAS for $6 \mathrm{~h}$, cells $\left(1 \times 10^{6}\right)$ were collected and rinsed once with $10 \mathrm{mM}$ Tris- $\mathrm{HCl}$ buffer, $\mathrm{pH} 8.7$, containing $3 \mathrm{mM} \mathrm{MgCl}_{2}$ and $2 \mathrm{mM} \mathrm{2-}$ mercaptoethanol then dissolved in $50 \mathrm{mM}$ Tris- $\mathrm{HCl}$ buffer, $\mathrm{pH} 7.8$, containing $10 \mathrm{mM}$ EDTA, $0.5 \%$ sodium lauroyl sarcocinate, and 1 $\mathrm{mg} / \mathrm{ml}$ proteinase $\mathrm{K}$. After incubation at $50^{\circ} \mathrm{C}$ for $30 \mathrm{~min}$, RNase A was added at a concentration of $0.5 \mathrm{mg} / \mathrm{ml}$ and the mixture further incubated at $50^{\circ} \mathrm{C}$ for $15 \mathrm{~min}$. Lysates were mixed with an equal volume of loading buffer containing $89 \mathrm{mM}$ Tris, $\mathrm{pH} 8.4,2.5 \mathrm{mM}$ EDTA, and $89 \mathrm{mM}$ boric acid (TBE), with $20 \%$ glycerol, and $0.01 \%$ bromophenol blue. Samples were electrophoresed on $2 \%$ agarose gels in TBE containing $0.5 \mathrm{mg} / \mathrm{ml}$ ethidium bromide.

Western blot analysis. After incubation in the presence or absence of $50 \mu \mathrm{M}$ caspase inhibitors (z-Asp- $\mathrm{CH}_{2}-\mathrm{DCB}$, Ac-DNLD-CHO, Ac-DQTD-CHO, and Ac-VEID-CHO) for $1 \mathrm{~h}$ and further incubation with $0.5 \mu \mathrm{g} / \mathrm{ml}$ anti-FAS for $4 \mathrm{~h}$, cells $\left(2 \times 10^{6}\right)$ were collected and protein was extracted in lysis buffer [50 mM HEPES (pH 7.5), $150 \mathrm{mM} \mathrm{NaCl}, 10 \%$ glycerol, $1 \%$ Triton-X, $1.5 \mathrm{mM}$ $\mathrm{MgCl}_{2}, 1 \mathrm{mM}$ EGTA, $1 \mathrm{mM}$ sodium orthovanadate, $1 \%$ protease inhibitor cocktail (Sigma-Aldrich, St. Louis, MO, USA)] concentration determined by the bicinchoninic acid protein assay, and $20 \mu \mathrm{g}$ of protein per sample was subjected to $15 \%$ polyacrylamide gel electrophoresis then transferred to a 
Structure of effector caspases
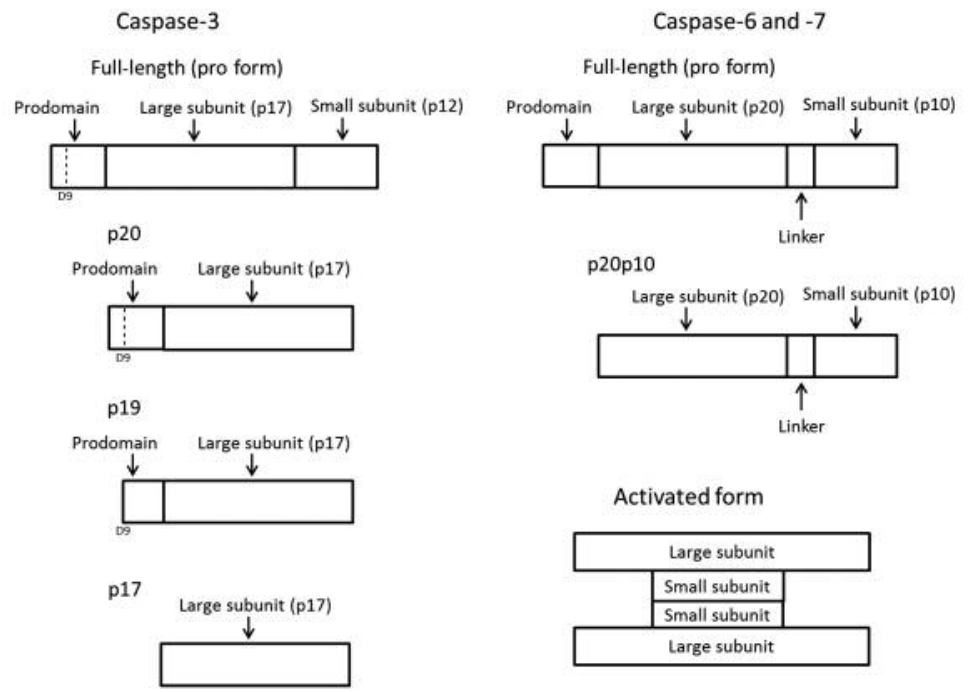

p20p10

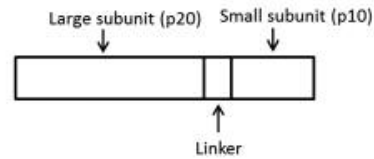

Activated form

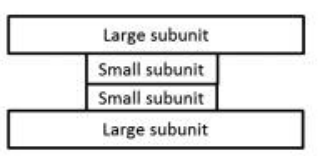

Figure 1. Caspases are composed of pro-domain, large subunit and small subunit. Large and small subunits are connected by linker of caspase-6 and -7 of effector caspases but not caspase-3. Activated caspase forms heterotetramer of large and small subunits.

polyvinylidene difluoride membrane. The membranes were blocked by $3 \%$ skimmed milk for $1 \mathrm{~h}$ and then incubated with primary antibodies at $4^{\circ} \mathrm{C}$ overnight. The primary antibodies used were: caspase-3 (1:1,000; Santa Cruz Biotechnology), caspase-7, caspase6 , and $\beta$-Actin $(1: 1,000$; Cell Signaling Technology, Danvers, MA, USA). After washing with $0.1 \%$ Tween 20-PBS, the membranes were incubated with horseradish peroxidase-conjugated goat anti-rabbit IgG secondary antibody for $60 \mathrm{~min}$ at room temperature. The membranes were finally visualized with enhanced chemiluminescence reagents (Pierce ECL Western Blotting Substrate; Thermo Scientific) following exposure at $1 \mathrm{~min}$ or $3 \mathrm{~min}$. Relative density of bands was quantified using ImageJ (National Institutes of Health, Bethesda, MD, USA).

Statistical analysis. All statistical analyses were performed using Student's $t$-test. Significance was established at the $p<0.05$ level.

\section{Results}

7A6 localization to mitochondria during apoptosis was inhibited by pan-caspase inhibitor z-Asp-CH$-\mathrm{H}_{2}-\mathrm{DCB}$. 7A6 is known to localize to mitochondria from cytosol during apoptosis $(16,18)$. The purpose of this experiment was to elucidate the dependence of 7A6 localization to mitochondria on caspases. 7A6 localization to mitochondria during apoptosis was confirmed by confocal microscopy and the mitochondrial localization of 7A6 was quantified by FCM. We first treated Jurkat cells with antiFAS in order to induce apoptosis. Apoptosis was confirmed by DNA degradation assay by agarose gel electrophoresis. Anti-FAS treatment degraded DNA to a ladder form, and this degradation was significantly inhibited by pan-caspase inhibitor $\mathrm{z}-\mathrm{Asp}-\mathrm{CH}_{2}-$
DCB (Figure 2A). Treatment with anti-FAS activated caspase-8, an initiator caspase, thereby activating effector caspase-3 and inducing apoptosis. We found that caspase- 8 and -3 were cleaved by anti-FAS treatment, and this cleavage was inhibited by z-Asp$\mathrm{CH}_{2}$-DCB (Figure 2B). We next measured the distribution of mitochondrial membrane antigen 7A6 with the use of specific antibody Apo2.7. Figure 2C shows the confocal laser scanning microscopy analysis of digitonin-permeabilized Jurkat cells stained with PC5-labeled Apo2.7 (red fluorescence) and mitochondria-specific staining reagent Mitotracker green (green fluorescence). 7A6 was localized to mitochondria by anti-FAS treatment. However, in healthy cells, 7A6 was not detected. Since this experiment was performed without fixing of the cells, outflow of cytosolic protein such as 7A6 occurred by cell membrane permeabilization and mitochondria-bound 7A6 was only detected (18). Furthermore, we used FCM to analyze 7A6 localization to mitochondria, and found that 7A6 was significantly increased by apoptosis induced by anti-FAS but reduced by pan-caspase inhibitor z-Asp- $\mathrm{CH}_{2}$-DCB (Figure 2D).

Caspase activation assay in anti-FAS-induced apoptosis. As shown above and in our previous study, pan-caspase inhibition reduces 7A6 localization to mitochondria during apoptotic induction (18). We also determined that the caspase-3 inhibitor, Ac-DEVD-CHO, could reduce the appearance of 7A6 (18). However, Ac-DEVD-CHO also inhibits other effector caspases, caspase-6 and -7. Thus, AcDEVD-CHO is a less specific caspase-3 inhibitor, and we were unable to completely estimate which effector caspase 


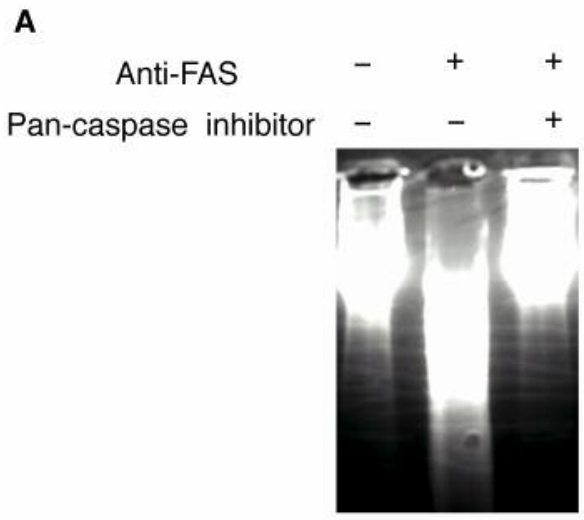

B

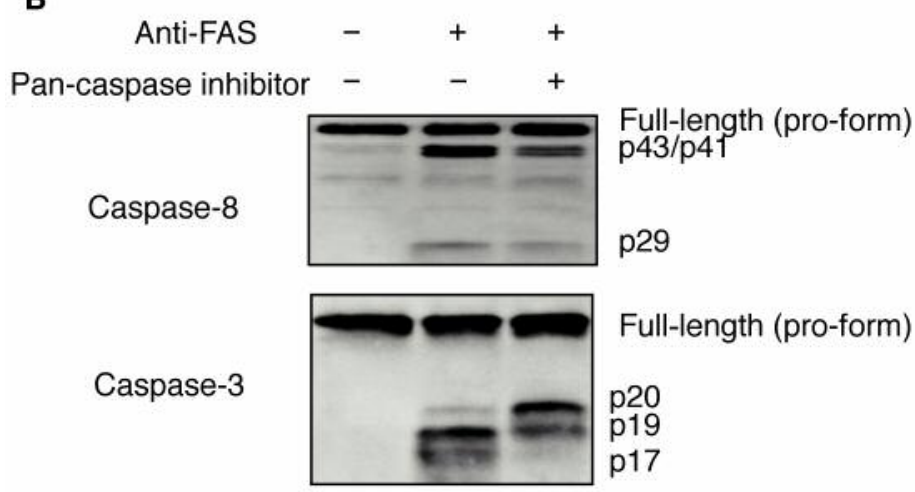

C Mitochondria 7A6 antigen Merge

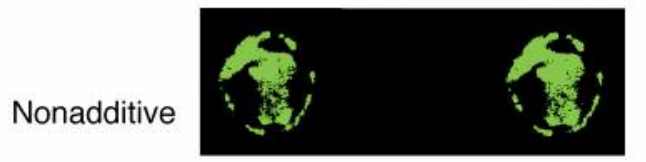

Anti-FAS

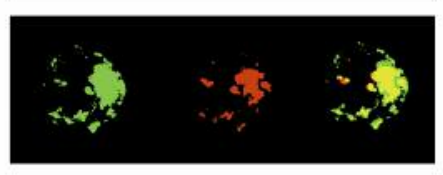

D

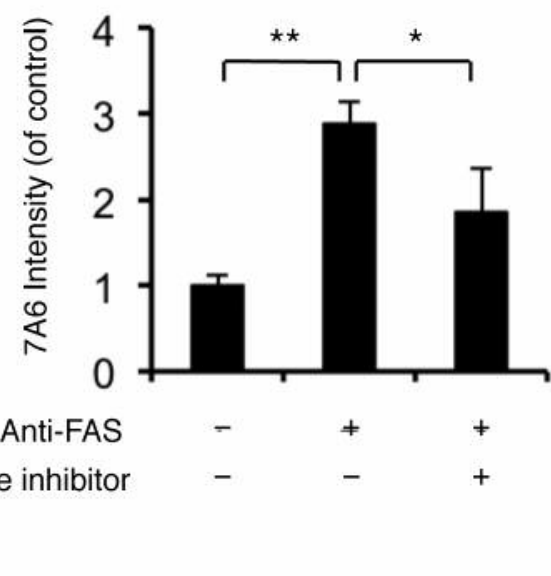

Figure 2. Anti-FAS induces $7 A 6$ localization to mitochondria, and pan-caspase inhibitor benzyloxycarbonyl-Asp-2,6-dichlorobenzoyloxymethylketone $\left(z-A s p-C_{2}-D C B\right)$ reduces $7 A 6$ localization in Jurkat cells. Jurkat cells were incubated in the presence or absence of $50 \mu M$ pan-caspase inhibitor $z$-Asp- $\mathrm{CH}_{2}-\mathrm{DCB}$ for $1 \mathrm{~h}$ and further incubated with $0.5 \mu \mathrm{g} / \mathrm{ml}$ anti-FAS for $4 \mathrm{~h}(\mathrm{~B})$ or $6 \mathrm{~h}(\mathrm{~A})$. A: Cellular DNA was collected and a ladder form was detected by agarose electrophoresis. B: Cellular protein was dissolved and caspase-8 and caspase-3 cleavage was detected by western blotting. C: Jurkat cells were treated with/without $0.5 \mu \mathrm{g} / \mathrm{ml}$ anti-FAS for $37^{\circ} \mathrm{C}$ at $6 \mathrm{~h}$. Mitochondria were stained with $100 \mathrm{nM}$ MitoTracker Green (green) at $37^{\circ} \mathrm{C}$ for $1 \mathrm{~h}$. After digitonin permeabilization, 7A6 was then stained with Apo2.7-phycoerythrin-cyanin 5.1 (PC5) (red) at $37^{\circ} \mathrm{C}$ for $1 \mathrm{~h}$ and cells observed under a confocal microscope. D: Jurkat cells were induced with/without $0.5 \mu \mathrm{g} / \mathrm{ml}$ anti-FAS antibody at $37^{\circ} \mathrm{C}$ for 6 h. After digitonin permeabilization, cells were stained with Apo2.7- PC5 at $37^{\circ} \mathrm{C}$ for $1 \mathrm{~h}$ and detected by flow cytometry. Data represent the average $\pm S D$ from three independent experiments. ${ }^{*} p<0.05$ and ${ }^{* *} p<0.005$ compared to the anti-FAS treatment group.

is involved in 7A6 localization. Therefore, we decided to use more highly specific effector caspase inhibitors other than Ac-DEVD-CHO. We used caspase-3-specific inhibitor AcDNLD-CHO, caspase-7/3-specific inhibitor Ac-DQTD-CHO (since there is no caspase-7 specific inhibitor), and caspase6-specific inhibitor Ac-VEID-CHO to estimate which caspases are involved in 7A6 localization.

Caspases are constructed of a pro-form, pro-domain, large subunit, and small subunit, and are cleaved upon activation (Figure 1). We used antibodies to caspase recognizing a large subunit so that only a small subunit or a pro-domain is not detected by western blotting.

Jurkat cells were treated with anti-FAS and effector procaspase cleavage was determined by western blotting. When anti-FAS was the sole treatment, caspase- 3 cleaved to p20, p19, and p17 (Figures 2B and 3). Caspase-7 and -6 were cleaved to large subunit (p20), small subunit (p10) (p20p10) and to $\mathrm{p} 20$, respectively. When cells were pre-incubated with pan-caspase inhibitor z-Asp- $\mathrm{CH}_{2}-\mathrm{DCB}$, caspase-3 partially cleaved p20 and the p19 form appeared (Figure 3); caspase-7 partially cleaved to p20p10 but not to p20; and caspase-6 inhibited expression of p20p10 and p20. When cells were preincubated with caspase-3 inhibitor Ac-DNLD-CHO or caspase-7/3 inhibitor Ac-DQTD-CHO, caspase-3 and -7 cleavage were similar to the case when pan-caspase inhibitor was used, however, Ac-DQTD-CHO inhibited p20p10 of caspase-7 more clearly than Ac-DNLD-CHO. Moreover, these inhibitors did not inhibit the appearance of p20p10 caspase-6. Caspase-6 inhibitor Ac-VEID-CHO did not affect anti-FASinduced cleavage of caspase- 3 and -7 . It did significantly 


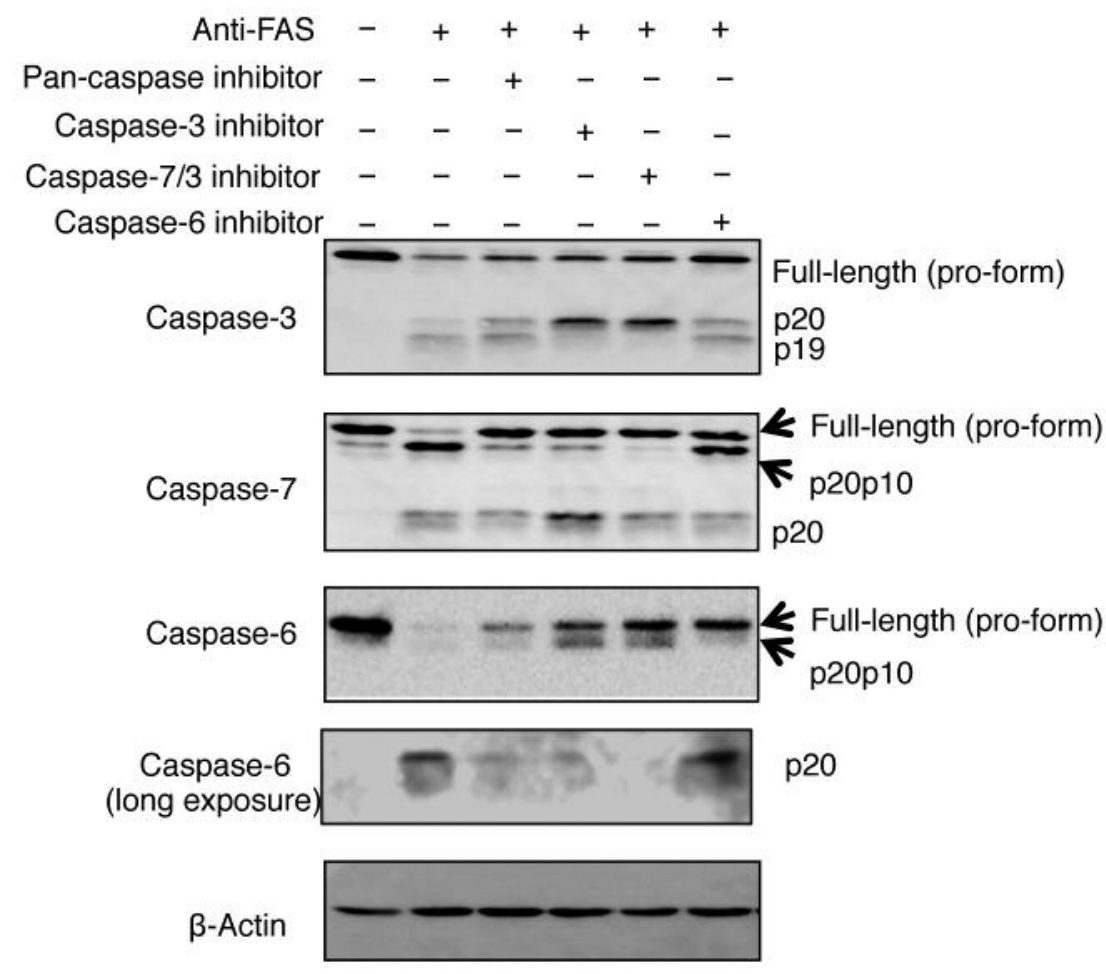

Figure 3. Anti-FAS induced effector caspase cleavage in Jurkat cells. Jurkat cells were incubated in the presence or absence of $50 \mu M$ caspase inhibitor for $1 \mathrm{~h}$ and further incubated with $0.5 \mu \mathrm{g} / \mathrm{ml}$ anti-FAS for $4 \mathrm{~h}$. Effector caspases and $\beta$-actin were detected by western blotting.

inhibit cleavage of p20p10 caspase- 6 but not that of p20 caspase-6 (Figure 3). These results suggest that the specific effector caspase inhibitors we used in this study only inhibit single individual caspases under our experiment conditions.

7A6 localization is correlated with the presence of p20p10 caspase-6. Next, we inhibited each caspase by its specific inhibitor and detected 7A6 localization to mitochondria using FCM. As shown in Figure 3A, caspase-3-specific inhibitor treatment hardly inhibited 7A6 appearance. Treatment with caspase-7/3-specific inhibitor suppressed 7A6 localization more than caspase- 3 inhibitor did. However, the most efficient suppression occurred by the use caspase-6-specific inhibitor (Figure 4A). The data for inhibition of 7A6 appearance by caspase inhibitors seemed to be similar to that in Figure 3 showing the level of FAS-induced p20p10 caspase- 6 inhibited by caspase inhibitors. Figure 4B shows quantification of p20p10/full-length caspase- 6 ratio shown in Figure 3 and compares the results with those using a nonadditive control (Figure 4B). Comparing the graphs in Figure $4 \mathrm{~A}$ and $\mathrm{B}$, we can see that the levels of mitochondrial 7A6 localization and p20p10 caspase- 6 are correlated, suggesting that caspase- 6 activation is involved in 7A6 localization to mitochondria.
Caspase-6 knockdown by specific siRNA reduces the appearance of 7A6. 7A6 localization was reduced the most by caspase- 6 inhibitor. Therefore, we determined the mechanism underlying caspase- 6 regulation of 7A6 localization. As shown in Figure 5A, caspase-6 expression was reduced by the transfection of specific caspase- 6 siRNA in Jurkat cells. Appearance of FAS-induced 7A6 was reduced by the down-regulation of caspase- 6 expression (Figure 5B). These results indicate that caspase- 6 activation accompanies 7A6 localization to mitochondria.

\section{Discussion}

In the present study, we demonstrated that FAS-induced apoptosis accompanying 7A6 localization to mitochondria was inhibited mainly by one of the inhibitors of effector caspase-6. Moreover, caspase- 6 knockdown also inhibited 7A6 localization to mitochondria, suggesting that caspase-6 activation is a key to 7A6 mitochondrial localization. In a previous study, 7A6 localization to mitochondria during apoptosis was inhibited by Ac-DEVD-CHO, a caspase-3 inhibitor (18). However, Ac-DEVD-CHO inhibited other effector caspases in that study. Therefore, in the present study we used caspase-3 inhibitor Ac-DNLD-CHO, caspase- 
A

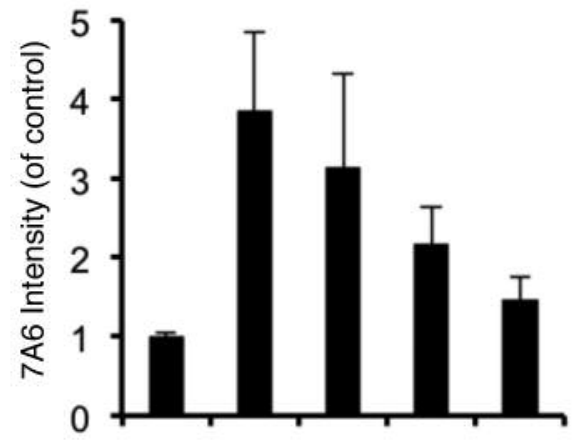

Anti-FAS

Caspase-3 inhibitor

Caspase-7/3 inhibitor

Caspase-6 inhibitor

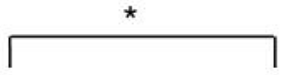

B
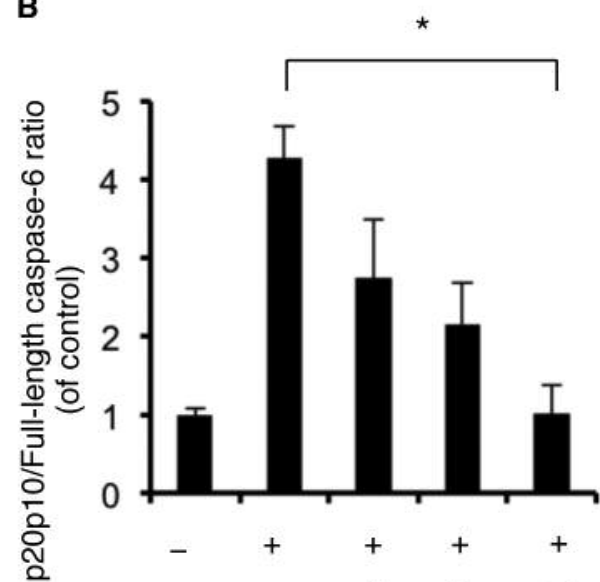

$-\quad-\quad+\quad-\quad-$

$\begin{array}{lllll}- & - & - & +\end{array}$

- $\quad-\quad-\quad-\quad+$

Figure 4. 7A6 detection is correlated with p20p10 caspase-6 expression. Jurkat cells were incubated in the presence or absence of $50 \mu M$ caspase inhibitor for $1 \mathrm{~h}$ and further incubated with $0.5 \mu \mathrm{g} / \mathrm{ml}$ anti-FAS for $6 \mathrm{~h}$. A: 7A6 was detected by flow cytometry. B: p20p10 caspase-6 was measured from the blots shown in Figure 2 by the Image $J$ software. Data represent the average $\pm S D$ from three independent experiments. ${ }^{*} p<0.05$ compared to the anti-FAS treatment group.

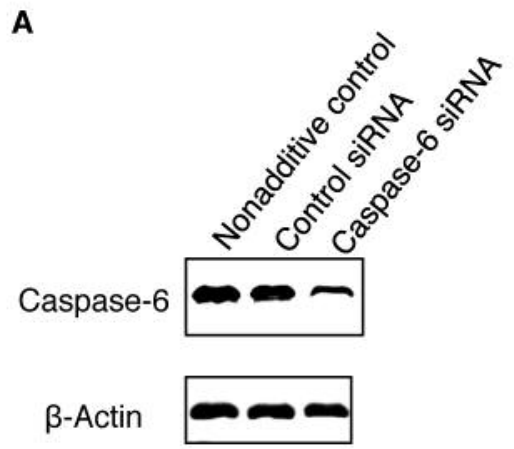

B

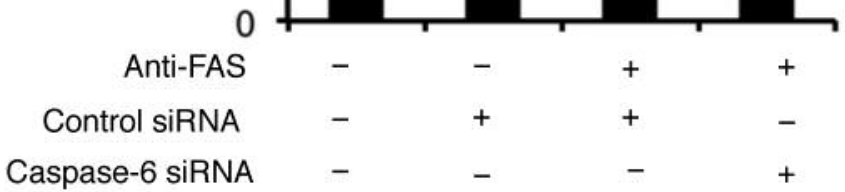

Figure 5. Caspase-6 down-regulation by specific siRNA reduced the appearance of 7A6. A: Caspase-6 targeting siRNA and negative control siRNA were transfected into Jurkat cells. Protein samples were collected at 48 h after siRNA transfection. Caspase-6 knockdown was confirmed by western blotting. B: Jurkat cells were incubated with $0.5 \mu \mathrm{g} / \mathrm{ml}$ anti-FAS for $6 \mathrm{~h} .7 A 6$ was detected by flow cytometry. Data represent the average $\pm S D$ from three independent experiments.

7/3 inhibitor Ac-DQTD-CHO, and caspase-6 inhibitor AcVEID-CHO to specifically inhibit effector caspases.

Caspase-3 recognizes and cleaves D179 of caspase-6 for activation $(2,21)$. However, activation of caspase- 6 has also been reported to occur in caspase-3-deficient cells (22). Western blotting (Figure 3) revealed that during caspase-3 inhibition, p20 caspase- 6 was not expressed but p20p10 caspase- 6 was (Figure 3). Conversely, under caspase-6 inhibition, p20p10 caspase- 6 was not expressed but p20 caspase- 6 was. Activated forms of caspases are considered heterotetramers of large and 
small subunits (23). However, p20p10 caspase-6 also has enzymatic activity. In fact, p20p10 caspase- 6 has such activity in vivo (2). Therefore, when caspase-3 inhibitor and caspase-7/3 were used to inhibit caspase, it was considered that p20p10 caspase- 6 would not be inhibited and therefore 7A6 localization to mitochondria would not be inhibited sufficiently. Furthermore, the present results suggest that caspase- 6 inhibitor inhibits the expression of active p20p10 caspase- 6 and directly inhibits the expressed activated form of caspase- 6 (p20 and p10 heterotetramer). These results in turn suggest that 7A6 correlates with p20p10 caspase- 6 expression and is regulated by caspase6. Moreover, in caspase- 6 down-regulation by siRNA (Figure $5 \mathrm{~A}$ ), we also confirmed a decrease in caspase- 6 expression and a decrease in 7A6 localization to mitochondria (Figure 5B). These results suggest that 7A6 localization to mitochondria is regulated by caspase- 6 .

The substrate of caspase- 6 is well known to be lamin $\mathrm{A} / \mathrm{C}$, the degradation of which is used as an indicator of caspase- 6 activation (24). Although lamin $\mathrm{A} / \mathrm{C}$ is a constitutive protein of the nuclear membrane, we have confirmed by confocal microscopy that lamin $\mathrm{A} / \mathrm{C}$ is not co-localized with 7A6 (data not shown). Herpesvirus-associated ubiquitin-specific protease, kinesin 5B, guanine nucleotide-exchange protein 100, serologically defined colon cancer antigen 3 , and zinc finger CCHC-type containing 11 were also shown to be substrates during caspase- 6 apoptosis, but none of the proteins were reported to be localized to mitochondria (25-27), suggesting that 7A6 is a new candidate substrate for caspase-6.

We demonstrated that caspase- 6 regulates the localization of 7A6. Caspase-6 is an effector caspase and activates downstream from mitochondria during apoptosis. However, there are many substrates for caspase- 6 , and there is a possibility that 7A6 might affect mitochondria. Moreover, it is unknown whether caspase-6 acts on 7A6 directly or indirectly. Therefore, it will be necessary to identify the precise action of 7A6 and to establish the meaning of 7A6 localization to mitochondria during apoptosis.

\section{References}

1 Chai J and Shi Y: Apoptosome and inflammasome: conserved machineries for caspase activation. Natl Sci Rev 1: 101-118, 2014

2 Klaiman G, Champagne N and LeBlanc AC: Self-activation of Caspase- 6 in vitro and in vivo: Caspase- 6 activation does not induce cell death in HEK293T cells. Biochim Biophys Acta 1793: 592-601, 2008.

3 Lamkanfi $\mathrm{M}$ and Kanneganti TD: Caspase-7: a protease involved in apoptosis and inflammation. J Biochem Cell Biol 42: 21-24, 2010

4 Fernandes-Alnemri T, Armstrong RC, Krebs J, Srinivasula SM, Wang L, Bullrich F, Fritz LC, Trapani JA, Tomaselli KJ, Litwack $\mathrm{G}$ and Alnemri ES: In vitro activation of $\mathrm{CPP} 32$ and $\mathrm{MCH} 3$ by $\mathrm{MCH} 4$, a novel human apoptotic cysteine protease containing two FADD-like domains. Proc Natl Acad Sci USA 93: 7464-7469, 1996.
5 Park YH, Jeong MS and Jang SB: Structural insights of homotypic interaction domains in the ligand-receptor signal transduction of tumor necrosis factor (TNF). BMB Rep 49: 159$166,2016$.

6 Toyoshima F, Moriguchi $\mathrm{T}$ and Nishida E: FAS induces cytoplasmic apoptotic responses and activation of the MKK7JNK/SAPK and MKK6-p38 pathways independent of CPP32like proteases. J Cell Biol 139: 1005-1015, 1997.

7 Slee EA, Adrain C and Martin SJ: Executioner caspase-3, -6, and -7 perform distinct, non-redundant roles during the demolition phase of apoptosis. J Biol Chem 276: 7320-7326, 2001.

8 Cowling V and Downward J: Caspase-6 is the direct activator of caspase- 8 in the cytochrome c-induced apoptosis pathway: absolute requirement for removal of caspase- 6 prodomain. Cell Death Differ 9: 1046-1056, 2002.

$9 \mathrm{Li} \mathrm{H}, \mathrm{Zhu} \mathrm{H}, \mathrm{Xu} \mathrm{CJ}$ and Yuan J: Cleavage of BID by caspase 8 mediates the mitochondrial damage in the FAS pathway of apoptosis. Cell 94: 491-501, 1998.

10 Hiura TS, Li N, Kaplan R, Horwitz M and Seagrave JC and Nel AE: The role of a mitochondrial pathway in the induction of apoptosis by chemicals extracted from diesel exhaust particles. J Immunol 165: 2703-11, 2000.

11 Okinaga T, Kasai H, Tsujisawa $\mathrm{T}$ and Nishihana T: Role of caspases in clearage of lamin $\mathrm{A} / \mathrm{C}$ and PARP during apoptosis in macrophages infected with a periodontopathic bacterium. J Med Microbiol 56: 1399-1404, 2007.

12 Li P, Nijhawan D, Budihardjo I, Srinivasula SM, Ahmad M, Alnemri ES and Wang X: Cytochrome c and dATP-dependent formation of APAF-1/caspase-9 complex initiates an apoptotic protease cascade. Cell 91: 479-489, 1997.

13 Suzuki Y, Imai Y, Nakayama H, Takahashi K, Takio K and Takahashi R: A serine protease, HTRA2, is released from the mitochondria and interacts with XIAP, inducing cell death. Mol Cell 8: 613-621, 2001.

14 Candé C, Cohen I, Daugas E, Ravagnan L, Larochette N, Zamzami $\mathrm{N}$ and Kroemer G: Apoptosis-inducing factor (AIF): a novel caspase-independent death effector released from mitochondria. Biochimie 84: 215-222, 2002.

$15 \mathrm{Li} \mathrm{LY}$, Luo $\mathrm{X}$ and Wang $\mathrm{X}$ : Endonuclease $\mathrm{G}$ is an apoptotic DNase when released from mitochondria. Nature 412: 95-99, 2001.

16 Zhang C, Ao Z, Seth A and Schlossman SF: A mitochondrial membrane protein defined by a novel monoclonal antibody is preferentially detected in apoptotic cells. J Immunol 157: 39803987, 1996.

17 Koester SK, Schlossman SF, Zhang C, Decker SJ and Bolton WE: APO2.7 defines a shared apoptotic-necrotic pathway in a breast tumor hypoxia model. Cytometry 33: 324-332, 1998.

18 Nagahara Y, Tanaka M and Shinomiya T: Mechanism of mitochondrial 7A6 antigen exposure triggered by distinct apoptotic pathways: involvement of caspases. Cytometry A 71: 232-241, 2007.

19 Kuželová K, Grebeňová D and Brodská B: Dose-dependent effects of the caspase inhibitor Q-VD-OPh on different apoptosis-related processes. J Cell Biochem 112: 3334-3342, 2011.

20 Chéreau D, Kodandapani L, Tomaselli KJ, Spada AP and Wu JC: Structural and functional analysis of caspase active sites. Biochemistry 42: 4151-4160, 2003. 
21 Wang XJ, Cao Q, Liu X, Wang KT, Mi W, Zhang Y, Li LF, LeBlanc AC and Su XD: Crystal structures of human caspase 6 reveal a new mechanism for intramolecular cleavage selfactivation. EMBO Rep 11: 841-847, 2010.

22 Zheng N, Zhang P, Huang H, Liu W, Hayashi T, Zang L, Zhang Y, Liu L, Xia M, Tashiro S, Onodera S and Ikejima T: ER $\alpha$ down-regulation plays a key role in silibinin-induced autophagy and apoptosis in human breast cancer MCF-7 cells. J Pharmacol Sci 128: 97-107, 2015.

23 Fuentes-Prior P and Salvesen GS: The protein structures that shape caspase activity, specificity, activation and inhibition. Biochem J 384: 201-232, 2004.

24 Mintzer R, Ramaswamy S, Shah K, Hannoush RN, Pozniak CD, Cohen F, Zhao X, Plise E, Lewcock JW and Heise CE: A wholecell assay to measure caspase- 6 activity by detecting cleavage of lamin A/C. PLoS One 7: e30376, 2012.
25 Cho JH, Lee PY, Son WC, Chi SW, Park BC, Kim JH and Park SG: Identification of the novel substrates for caspase-6 in apoptosis using proteomic approaches. BMB Rep 46: 588-593, 2013.

26 Yuan J, Luo K, Zhang L, Cheville JC and Lou Z: USP10 regulates p53 localization and stability by deubiquitinating p53. Cell 140: 384-396, 2010.

27 Blahna MT, Jones MR, Quinton LJ, Matsuura KY and Mizgerd JP: Terminal uridyltransferase enzyme ZCCHC11 promotes cell proliferation independent of its uridyltransferase activity. J Biol Chem 286: 42381-42389, 2011.

Received February 9, 2017

Revised March 8, 2017

Accepted March 10, 2017 